\title{
A multiperversity generalization of intersection homology
}

\author{
Greg Friedman* Gil Kalai ${ }^{\dagger}$
}

November 28, 2005

\begin{abstract}
We define a generalization of intersection homology based on considering a set of perversities rather than a single perversity and explore some of its properties. The question whether these invariants are independent from the stratification is left open. Some steps in this direction are made following the proof by King of the topological incariance of singular intersection homology groups.
\end{abstract}

2000 Mathematics Subject Classification: 55N33, 57N80

Keywords: Intersection homology, perversity, pseudomanifold, manifold stratified space

\section{Introduction}

Intersection homology groups $I H_{*}^{\bar{p}}(X)$ for stratified spaces $X$ were discovered by Goresky and MacPherson in $[3,4]$. These groups depend on certain sequences of numbers $\bar{p}=$ $(\bar{p}(0), \bar{p}(1), \ldots)$ which are called perversities. These sequences satisfy the following simple properties: $\bar{p}(0)=\bar{p}(1)=\bar{p}(2)=0$ and $\bar{p}(i) \leq \bar{p}(i+1) \leq \bar{p}(i)+1$. Roughly speaking, these perversities describe how simplices are allowed to intersect the singular part of $X$. Goresky and MacPherson proved two remarkable basic results for these invariants for the case that $X$ is a pseudomanifold. The first is that intersection homology groups are topological invariants and, in particular, do not depend on the stratification of $X$. From this it follows that for the case that $X$ is a compact $n$-manifold, $I H_{i}^{\bar{p}}(X)$ coincide with the usual homology $H_{i}(X)$ for every perversity and every stratification. The second is that intersection homology of $n$-dimensional pseudomanifolds satisfies a certain form of duality

$$
I H_{i}^{\bar{p}}(X)=I H_{n-i}^{\hat{p}}(X),
$$

where and $\hat{p}$ is the dual perversity of the perversity $\bar{p}$, defined by $\hat{p}(i)+\bar{p}(i)=i-2$ for every $i$.

*Vanderbilt University

${ }^{\dagger}$ Hebrew University and Yale University 
When $X$ is a complex algebraic variety, $X$ can be stratified with only even dimensional strata and it follows that for the middle perversity $\bar{p}$ defined by $\bar{p}(i)=[(i-1) / 2]$,

$$
I H_{i}^{\bar{p}}(X)=I H_{n-i}^{\bar{p}}(X) \text {. }
$$

While general intersection homology groups are remarkable invariants of general singular spaces and pseudomanifolds, it is fair to say that the case of middle perversity intersection homology of algebraic varieties accounts for most applications. Those range from algebraic geometry and representation theory to the combinatorial theory of convex polytopes.

In this paper we explore an extension of intersection homology groups $I H_{*}^{J}(X)$ when a single perversity is replaced by a whole set $J$ of perversities. The purpose of this study is to obtain further interesting homology-like invariants for singular spaces. As intersection homology (in middle perversity) leads to remarkable combinatorial invariants of convex polytopes, hoping to find (eventually) additional combinatorial invariants for polytopes was another motivation.

Our analysis of these invariants follows a paper by Henry King [5]. King considered singular intersection homology theory and presented a direct proof (avoiding Deligne's sheaf) that singular intersection homology is a topological invariant of $X$. In the setting of singular simplices, topological invariance is equivalent to showing that the invariants do not depend on the stratification. King's proof of the topological invariance is based on several properties of intersection homology. It requires extensions of several basic properties of homology and, in addition, an understanding of intersection homology of cones over spaces and of products of spaces with manifolds. King's Theorem 9 gives an inductive proof of topological invariance and the steps in his argument can be regarded as a "road map" for such a proof in more general cases. King's Theorem 10 asserts that invariants of singular spaces with certain natural properties are equivalent if they have the same behavior with respect to forming a cone over a space. The behavior of intersection homology with respect to forming a cone is very simple and thus King's result can be regarded as an invitation for further invariants

For a stratified space $X$ let $c X$ be an open cone over $X$ (with the natural stratification). A main result of this paper is a "cone formula", namely, a formula for $I H_{*}^{J}(c X)$. We also establish some basic properties such as Mayer-Vietoris and Kunneth theorems. Our cone formula is rather complicated and we cannot use it so far to prove that our invariants do not depend on the stratification. Proving (or disproving) topological invariance remains the main open problem. Another problem would be to understand if some form of duality holds in this greater generality.

\section{Definition and most basic properties}

All spaces are locally-conelike stratified spaces $X$ with skeleta

$$
X=X^{n} \supset X^{n-1} \supset \cdots \supset X^{0} \supset X^{-1}=\emptyset .
$$

We recall that this means that each $X^{i}-X^{i-1}$ is a topological $i$-dimensional manifold (Hausdorff, metrizable, and separable), and for each $x \in X^{i}-X^{i-1}, 0 \leq i \leq n-1$, there 
is a neighborhood of $x$ stratified homeomorphic to $\mathbb{R}^{i} \times c Y$, where $Y$ is a compact filtered space and $\mathbb{R}^{i} \times c Y$ is given the filtration induced by that of $Y$ (see [5]). Here $c Y$ is the open cone on $Y$. Throughout, $c X$ will denote the open cone if $X$ is a space, and $c \xi$ will denote the closed cone if $\xi$ is a chain.

Let $J=\left\{\bar{p}_{1}, \cdots, \bar{p}_{m}\right\}$ be a collection of traditional perversities, i.e. for each $j, \bar{p}_{j}(2)=0$ and $\bar{p}_{j}(k) \leq \bar{p}_{j}(k+1) \leq \bar{p}_{j}(k)+1$.

Recall ([5]) that a singular simplex $\sigma \in C_{i}(X ; G), \sigma: \Delta^{i} \rightarrow X$, is said to be $\bar{p}$ allowable if for each $k, \sigma^{-1}\left(X^{n-k}\right)$ is a subset of the $i-k+\bar{p}(k)$ skeleton of the model simplex $\Delta^{i} . I C_{*}^{\bar{p}}(X)$ is the complex of chains $\xi$ such that each simplex of $\xi$ and $\partial \xi$ (with non-zero coefficient) is allowable.

Let $I C_{i}^{J}(X ; G)$ be the subgroup of $C_{i}(X ; G)$ consisting of $i$-chains $\xi$ such that $\xi=\sum_{j} \xi_{\bar{p}_{j}}$ and $\partial \xi=\sum_{j} \zeta_{\bar{p}_{j}}$, where $\xi_{\bar{p}_{j}}$ and $\zeta_{\bar{p}_{j}}$ are $G$-linear combinations of $\bar{p}_{j}$ allowable simplices. In other words, each simplex of $\xi$ and $\partial \xi$ must be allowable with respect to some perversity in $J$. Note that $\zeta_{\bar{p}_{j}}$ is not necessarily equal to $\partial \xi_{\bar{p}_{j}}$. Let $I H_{*}^{J}(X ; G)$ be the homology groups of $I C_{*}^{J}(X ; G)$. We will generally omit the coefficient group $G$ to simplify notation.

Note that if $J$ contains only a single perversity $\bar{p}$, then $I H_{*}^{J}=I H_{*}^{\bar{p}}$. If $U$ is an open subset of $X$, then we define $I H_{*}^{J}(X, U)$ in the usual way.

Lemma 2.1 (Subdivision). Suppose $\xi$ is a cycle in $I C_{i}^{J}(X, U)$. Then $\xi$ is homologous to any subdivision $\xi^{\prime}$ of $\xi$.

Proof. The proof is essentially the same as that given in [1] for the case in which $J$ contains a single perversity - if $\sigma$ is $\bar{p}_{j}$ allowable for some $\bar{p}_{j}$, then each simplex in any subdivision of $\sigma$ is also $\bar{p}_{j}$ allowable, so $\xi^{\prime}$ is allowable. Furthermore, an allowable homology between $\xi$ and $\xi^{\prime}$ is constructed by suitably subdividing $\Delta \times[0,1]$ for each simplex $\sigma: \Delta \rightarrow X$ so that one end, say $\Delta \times 0$, remains unsubdivided, and the other end, $\Delta \times 1$, is subdivided as per $\xi^{\prime}$. These subdivisions can be chosen so that the chain determined by the composition of $\sigma$ with the projection of the subdivided $\Delta \times I$ to $\Delta$ is $\bar{p}_{j}$ allowable. Employing this process compatibly for each $\sigma$ and with the relevant coefficients provides the desired homology. More precise details can be found in [1]. The main point here is that we can maintain the decomposition of $\xi$ into pieces allowable with respect to different perversities.

\section{The cone formula}

In this section we find the formula for the multiperverse intersection homology of a cone. This formula plays a fundamental role in single perversity intersection homology theory.

Let $J(i, k)=\left\{\bar{p}_{j} \in J \mid 0 \leq i-(k+1)+\bar{p}_{j}(k+1)\right\}$. These are the perversities $\bar{p}$ such that $I C_{i}^{\bar{p}}(c X)$, where $X$ is a $k$-dimensional compact locally-conelike space, includes chains whose supports intersect the cone point $x$.

Lemma 3.1. Suppose $\xi$ is a cycle in $I C_{i}^{J}(c X)$, where $X$ is an $n$-dimensional compact locallyconelike space. Let $x$ denote the cone point of $c X$. Then $\xi$ is homologous $\left(i n I C_{*}^{J}(c X)\right)$ to a chain of the form $c \psi+\gamma \in I C_{i}^{J}(c X)$, where 
1. $|\gamma| \cap x=\emptyset$,

2. $\psi=-\partial \gamma,|\psi| \cap x=\emptyset, \psi \in I C_{i-1}^{J(i, n)}(c X-x)$, and $c \psi$ is the (closed) cone on $\psi$.

In particular, $\xi$ is homologous to a cycle whose simplices intersect $x$ at most at vertices.

Proof. We begin by subdividing $\xi$ and then breaking it up into pieces, one of which will be $\gamma$ and one of which we will modify to be $c \psi$. Let $U$ be an open neighborhood of the cone point $x \in c X$ that is disjoint from all simplices of $\xi$ whose supports do not intersect $x$. Let $V$ be $c X-x \cong X \times \mathbb{R}$. Let $\xi^{\prime}$ be a subdivision of $\xi$ that is $\{U, V\}$ small, so that $\xi^{\prime}$ is homologous to $\xi$ and $\xi^{\prime}=\eta+\gamma, \eta$ and $\gamma$ are allowable, $|\eta| \subset U$, and $|\gamma| \subset V$. Such subdivisions can be accomplished as per Proposition 2.9 of [1]. That proposition deals only with single perversity intersection homology, but the arguments work equally well (with obvious modifications) in our more general setting. Furthermore, by our choice of $U$, all simplices in $\eta$ will be simplices in a subdivision of those simplices of $\xi$ that intersect $x$. Since these simplices of $\xi$ must all be $J(i, n)$ allowable, so too will all $i$-simplices of $\eta$ be $J(i, n)$ allowable, as follows from the multiperversity generalization of the subdivision allowability arguments of Lemma 2.6 of [1]. Furthermore, we claim that $\partial \eta=-\partial \gamma$ is $J(i, n)$ allowable. This is a consequence of the actual chain map constructed in [1, Proposition 2.9] for breaking up chains into small pieces. The upshot is that one of the following scenarios holds for each $i-1$ simplex $\mu$ in $\eta$ :

1. $\mu$ has no $l$ face, $0 \leq l \leq i-1$, that is in the $l$ skeleton of any simplex of $\xi$ (in the sequel, we will refer to this condition as being "deeply embedded"). In this case, $\mu$ inherits the allowability of whichever simplex of $\xi$ it is contained in, and in this case such a simplex must be $J(i, n)$ allowable. (Note: for statements such as these about subsets and inclusions, we are really referring to the simplicial models upon which the singular simplices are built - see [1] for more careful statements).

2. $\mu$ is a simplex in a subdivision of $\partial \xi$. But since $\xi$ is a cycle, this is not possible.

We next claim that $\xi^{\prime}$ is homologous to $c(\partial \eta)+\gamma$. We check the details:

$c(\partial \eta)+\gamma$ is allowable: $\gamma$ is already allowable and since $\partial(c(\partial \eta)+\gamma)=\partial \eta+\partial \gamma=\partial(\eta+\gamma)=$ $\partial \xi^{\prime}=0$, to check allowability, we only need check the allowability of each simplex $c \sigma$ for $\sigma \in \partial \eta$. But each such $\sigma$ is in $I C_{i-1}^{J(i, n)}(c X-x)$. So each $\sigma$ is $\bar{p}$ allowable for some $\bar{p} \in J(i, n)$. But this implies that $c \sigma$ is allowable by the arguments in, e.g. King [5]. Geometrically, for $k<n+1,(c \sigma)^{-1}\left((c X)^{n+1-k}\right)$ intersects skeleton of $\Delta^{i}$ of one dimension greater than the skeleton containing $\sigma^{-1}(c X-x)$. However, $c \sigma$ is one dimension greater than $\sigma$, so the changes offset in the allowability formula. $c \sigma^{-1}(x)$ is a single vertex, and this is also allowable for each $\bar{p} \in J(i, n)$. Thus $c(\partial \eta)+\gamma$ is allowable.

To obtain the homology, we next observe that $c \eta$ is itself allowable. To see this, we again consider $c \sigma$ for each simplex $\sigma$ in $\eta$. Once again, for $k \neq n+1$, the dimension of the minimal skeleton of $\Delta^{i+1}$ containing $(c \sigma)^{-1}(c X)^{n+1-k}$ is at most one larger than the analogous dimension for $\sigma$, which is offset by the increase in dimension from $\sigma$ to $c \sigma$ in the allowability formula. For $\left(c \sigma^{-1}\right)(x)$, the same argument applies if $|\sigma| \cap x \neq \emptyset$, while if 
$|\sigma| \cap x=\emptyset,(c \sigma)^{-1}(x)$ will be a single vertex of $\Delta$, which once again is allowable for each $\bar{p} \in J(i, n)$.

Now let $H=c \eta+\gamma \times[0,1]$, where $\gamma \times[0,1]$ is induced by a subdivision of the trivial homotopy (which is also readily verified to preserve allowability as in [2]). Then $\partial H=$ $\eta-c(\partial \eta)+\gamma-\gamma=(\eta+\gamma)-(c(\partial \eta)+\gamma)$, demonstrating the desired homology.

Let $I C_{*}^{J(n)}(X)$ be the subcomplex of $I C_{*}^{J}(X)$ such that

$$
I C_{i}^{J(n)}(X)=\left\{\xi \in I C_{i}^{J}(X) \mid \xi \in I C_{i}^{J(i+1, n)}(X), \partial \xi \in I C_{i-1}^{J(i, n)}(X)\right\} .
$$

Note that the boundary map on this complex is well-defined as the restriction of the boundary map of $I C_{*}^{J}(X)$, and so we can define the homology groups $I H_{*}^{J(n)}(X)$. Let

$$
I C_{*}^{J / n}(X)=I C_{*}^{J}(X) / I C_{*}^{J(n)}(X)
$$

and let let $I H_{*}^{J / n}(X)$ be the corresponding homology groups.

Proposition 3.2 (Cone Formula). Let $X$ be an $n$-dimensional compact locally-conelike space. Then $I H_{i}^{J}(c X) \cong I H_{i}^{J / n}(X \times \mathbb{R}) \cong I H_{i}^{J / n}(X)$.

Proof. The second isomorphism is simply stratum-preserving homotopy equivalence or the Künneth theorem for products with manifolds (see Proposition 4.1, below, which is independent of the current proposition). So we focus on the first isomorphism. Throughout, we identify $X \times \mathbb{R}$ with $c X-x$, where $x$ is the cone point.

We define a homomorphism $f: I H_{i}^{J / n}(X \times \mathbb{R}) \rightarrow I H_{i}^{J}(c X)$ as follows. Let $\xi$ be a relative cycle representing an element of $I H_{i}^{J / n}(X \times \mathbb{R})$. So $\xi$ is $J$ allowable and $\partial \xi$ is $J(i, n)$ allowable (since $\partial^{2} \xi=0$, the condition that $\partial(\partial \xi)$ be $J(i-1, n)$ allowable is satisfied for free). Then let $f(\xi)$ be $\xi-c(\partial \xi)$ in $I C_{i}^{J}(c X)$. This chain is $J$ allowable precisely because $\partial \xi$ is $J(i, n)$ allowable, and it is an absolute cycle. We must show that $f$ is well defined as a map on homology. So let $\xi_{1}$ be another chain representing the same cycle as $\xi$ in $I H_{i}^{J / n}(X \times \mathbb{R})$. So there exists a $J$-allowable $i+1$ chain $\Xi$ such that $\partial \Xi=\xi-\xi_{1}+\phi$, where $\phi$ is $J(i+1, n)$ allowable and $\partial \phi$ is $J(i, n)$ allowable. Note that $\partial \phi=\partial \xi_{1}-\partial \xi$. Then $\Xi-c \phi$ is $J$-allowable (again because $\phi$ is $J(i+1, n)$ allowable and $\partial \phi$ is $J(i, n)$ allowable, so that coning on $\phi$ gives allowable chains). But now $\partial(\Xi-c \phi)=\partial \Xi-\partial(c \phi)=\partial \Xi-(\phi-c(\partial \phi))=$ $\xi-\xi_{1}+\phi-\left(\phi-c\left(\partial \xi_{1}-\partial \xi\right)\right)=\xi-c \partial \xi-\left(\xi_{1}-c \partial \xi_{1}\right)=f(\xi)-f\left(\xi_{1}\right)$. So $f$ is well-defined.

By Lemma 3.1, $f$ is a surjective homomorphism. To check that it is injective, suppose that $f(\xi)=\xi-c \partial \xi$ is the image of a chain representing a cycle in $I H_{i}^{J / n}(X \times \mathbb{R})$ and that $f(\xi)=0$ in $I H_{i}^{J}(c X)$. Then there is a $J$-allowable $i+1$ chain $\Xi$ such that $\partial \Xi=\xi-c \partial \xi$. We will split $\Xi$ into pieces. Let $V=c X-x$, and let $U$ be an open neighborhood of the cone point $x$ of $c X$ such that any simplex $\sigma$ in $\Xi$ with $x \notin|\sigma|$ satisfies $|\sigma| \cap U=\emptyset$. In particular, $|\xi| \cap U=\emptyset$. Now, as in the proof of Lemma 3.1, we may employ a multiperversity generalization of Proposition 2.9 of [1] to break $\Xi$ into $\Xi=\Xi_{U}+\Xi_{V}$, where $\left|\Xi_{U}\right| \subset U$, $\left|\Xi_{V}\right| \subset V$, and each of these chains is allowable. Again, the argument of [1] carries over because, by construction, each simplex of $\Xi_{U}$ and $\Xi_{V}$ is a simplex in a subdivision of $\Xi$, and each such simplex inherits the allowability of its parent simplex, and similarly each $i$ 
simplex of $\partial \Xi_{U}$ or $\partial \Xi_{V}$ is either deeply enough embedded in a simplex of $\Xi$ that it inherits its allowability or it is a subdivision simplex of a simplex already in $\partial \Xi$ and hence inherits allowability from that.

Now we consider $\Xi_{V}$ and $\partial \Xi_{V}$. Since $\partial\left(\Xi_{U}+\Xi_{V}\right)$ is just a subdivision of $\partial \Xi=\xi-c \partial \xi$ and since $|\xi| \cap U=\emptyset$, a subdivision of $\xi$ forms part of the boundary of $\Xi_{V}$. In fact, $\partial \Xi_{V}=\xi^{\prime}+\omega+\phi$, where $\xi^{\prime}$ is a subdivision of $\xi, \omega$ is a subchain of a subdivision of $-c \partial \xi$ and $\phi$ is a new bit of boundary that results from cutting $\Xi_{U}$ and $\Xi_{V}$ apart. But now since each $i$ simplex in $\omega$ lies in a subdivision of a simplex of $c \partial \xi$, which was $J(i, n)$ allowable, $\omega$ is $J(i, n)$ allowable. Similarly since $|\phi|$ must lie in $U \cap V \subset U$, each $i$-simplex of $\phi$ is in a subdivision of a simplex $\sigma$ of $\Xi$ whose support contains $x$. But each such simplex of $\Xi$ must be $J(i+1, n)$ allowable and so $\phi$ is $J(i+1, n)$ allowable (again, $\phi$ must be one of these "deeply embedded" subdivision simplices or else it would be part of the subdivision of $\partial \Xi=\xi-c \partial \xi)$. Thus $\xi^{\prime}=\partial \Xi_{V}-\omega-\phi$. As we've seen, $\omega$ is $J(i, n)$ allowable (and hence also $J(i+1, n)$ allowable since $J(i, n) \subset J(i+1, n))$, and $\phi$ is $J(i+1, n)$ allowable, and $\partial(\omega+\phi)=-\partial \xi^{\prime}$, which is $J(i, n)$ allowable as a subdivision of $\partial \xi$, which was $J(i, n)$ allowable to begin with. And so $\xi^{\prime}$ represents 0 in $I H_{i}^{J / n}(X \times \mathbb{R})$. But $\xi^{\prime}$ is a subdivision of $\xi$ and so by the Lemma 2.1, $\xi$ and $\xi^{\prime}$ are homologous. Thus $f$ is injective, and we are done.

Corollary 3.3. $I H_{i}^{J}(c Y, Y \times \mathbb{R}) \cong I H_{i-1}^{J(n)}(Y)$.

Proof. Consider the diagram

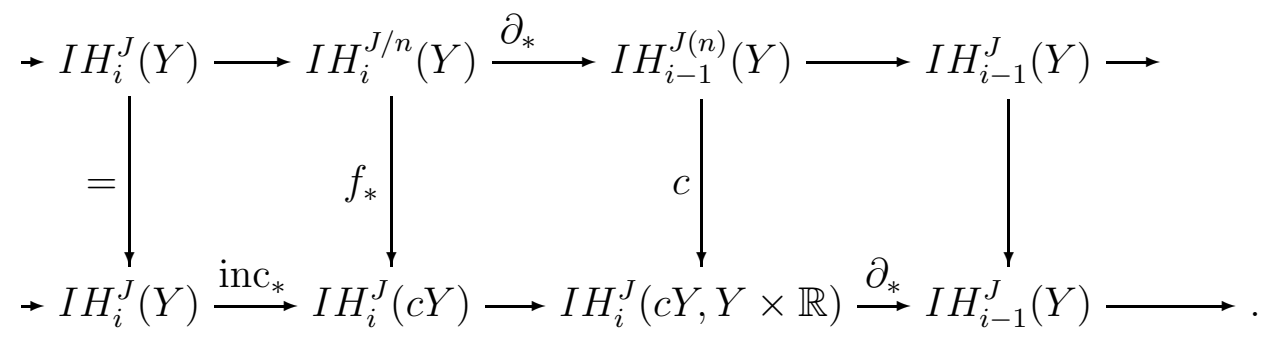

The first line is the exact sequence induced by the short exact sequence

$$
0 \longrightarrow I C_{i}^{J(n)}(Y) \longrightarrow I C_{i}^{J}(Y) \longrightarrow I H_{i}^{J / n}(Y) \longrightarrow 0
$$

The second line is the exact sequence induced by the short exact sequence

$$
0 \longrightarrow I C_{i}^{J}(Y \times \mathbb{R}) \longrightarrow I C_{i}^{J}(c Y) \longrightarrow I C_{i}^{J}(c Y, Y \times \mathbb{R}) \longrightarrow 0 .
$$

Once we show that the diagram commutes, the corollary will follow from Proposition 3.2 and the five lemma.

The map $f_{*}$ in the diagram is that of Proposition 3.2, while the map labeled $c$ takes the cone on chains.

The commutativity of the right square is straightforward, while that of the left and central squares follows from the definition of $f_{*}$ and some obvious diagram chasing.. 


\section{Some more basic properties}

Proposition 4.1 (Stratified homotopy invariance). $I H_{*}^{J}, I H_{*}^{J(n)}$, and $I H_{*}^{J / n}$ are invariant under stratum-preserving homotopy equivalences. In addition, any two chains that differ only by a stratum-preserving homotopy are homologous in any of these theories.

Proof. The proofs follow just as for the single perversity case; see [2]. The main point is that given a $\bar{p}$-allowable simplex in a filtered space, $\sigma: \Delta^{i} \rightarrow X$, each simplex in the chain induced by a subdivision of $\Delta^{i} \times[0,1]$ and a stratum-preserving homotopy $H: \Delta^{i} \times[0,1] \rightarrow X$ from $\sigma$ is also $\bar{p}$-allowable.

Corollary 4.2 (Künneth theorem for products with $\left.\mathbb{R}^{k}\right) . I H_{*}^{J}\left(X \times \mathbb{R}^{k}\right) \cong I H_{*}^{J}(X)$, $I H_{*}^{J(n)}\left(X \times \mathbb{R}^{k}\right) \cong I H_{*}^{J(n)}(X)$, and $I H_{*}^{J / n}\left(X \times \mathbb{R}^{k}\right) \cong I H_{*}^{J / n}(X)$.

Proposition 4.3. $I H_{*}^{J}$ admits Mayer-Vietoris sequences relating open sets $U, V, U \cap V$, and $U \cup V$. I $H_{*}^{J}$ also admit excision $I H_{*}^{J}(X, U) \cong I H_{*}^{J}(X-K, U-K)$, for $K$ closed in the open set $U$.

Proof. Once again, the proof is a straightforward generalization of that for single perversities; see [1]. The idea is to follow the proofs for ordinary singular homology as given in, e.g., [6]. The main technical point is the ability to break a chain into small pieces subordinate to some cover using subdivision. The main difficulty in intersection homology comes in ensuring that these pieces are allowable. There is no problem with the simplices in a chain, themselves, as subdivision preserves allowability. The difficulty is in making sure that the new boundaries created when pieces of the chain are broken apart are allowable. It is shown in [1] how to do this in such a way that the newly created boundary $i-1$ simplices inherit the same allowability as the $i$-simplices out of which they are carved. In the current case, the $J$ allowability of the original simplices thus ensures the $J$-allowability of the new pieces and their new boundaries after performing the construction of [1].

Note that $I H_{*}^{J(n)}$ and $I H_{*}^{J / n}$ generally will not admit Mayer-Vietoris properties and excision. This can be seen by considering the case where $J=\{\bar{p}\}$. In this case $I C_{*}^{J(n)}$ is a truncation of $I C_{*}^{\bar{p}}$, so that $I H_{*}^{J(n)}$ will equal $I H_{*}^{\bar{p}}$ for large $*$ and 0 below a certain cutoff dimension. Since $I H_{*}^{\bar{p}}$ itself admits Mayer-Vietoris sequences, $I H^{J(n)}$ will simply truncate that Mayer-Vietoris sequence, not preserving exactness in general. This failure can also be seen as a consequence of the fact that the allowability conditions on boundary simplices of chains in $I C_{*}^{J(n)}$ is more stringent than that on the simplices themselves. Thus the above arguments do not guarantee that the process of [1] yields allowable chains once we break a chain into pieces.

Proposition 4.4 (Künneth theorem for products with manifolds). If $X$ is a filtered space, $M$ is an unfiltered $k$-manifold, and $X \times M$ is given the product filtration, then $I H_{*}^{J}(X \times$ $M)=\left(\bigoplus_{p+q=*} I H_{p}^{J}(X) \otimes I H_{q}^{J}(M)\right) \oplus\left(\bigoplus_{p+q=*-1} I H_{p}^{J}(X) * I H_{q}^{J}(M)\right)$.

Proof. This property is proven in [5] for single perversity intersection homology by noting that it is a consequence of the existence of Mayer-Vietoris sequences and the Künneth theorem for products with $\mathbb{R}^{k}$. Since $I H_{*}^{J}$ also has these properties, the same proof applies. 


\section{Dimension relations}

Lemma 5.1. Let $Y$ be at-dimensional stratified space, and suppose $n \geq t$. Then $I H_{i}^{J(n)}(c Y)=$ 0 , and $I H_{i}^{J}(c Y) \cong I H_{i}^{J / n}(c Y)$.

Proof. The second statement follows from the first using the long exact sequence

$$
I H_{i}^{J(n)}(c Y) \longrightarrow I H_{i}^{J}(c Y) \longrightarrow I H_{i}^{J / n}(c Y) \longrightarrow I H_{i-1}^{J(n)}(c Y)
$$

To begin, we assume $t=n$.

Suppose $\xi$ represents a cycle in $I H_{i}^{J(n)}(c Y)$. Then each simplex in $\xi$ is allowable with respect to a perversity $\bar{p}$ such that $0 \leq i+1-(n+1)+\bar{p}(n+1)$. We claim that each simplex in $\bar{c} \xi$ is also allowable with respect to such a perversity, which would demonstrate that each cycle bounds. The argument is essentially that which occurs for the intersection homology of a cone with a single perversity once we have passed the critical dimension at which all intersection homology dies. A thorough treatment of that case can be found in [5] and [1]. We indicate the ideas here:

Suppose $\sigma$ is a simplex in $\xi$. If $\sigma^{-1}\left((c Y)^{n+1-k}\right), k \leq n$, lies in the $\ell$ skeleton of $\Delta^{i}$, then $(c \sigma)^{-1}\left((c Y)^{n+1-k}\right)$ lies in the $\ell+1$ skeleton of $\Delta^{i+1}$. Allowability of $\sigma$ implies that $\ell \leq i-k+\bar{p}(k)$ for some $\bar{p}$ in $J(i+1, n)$. This implies that $\ell+1 \leq i+1-k+\bar{p}(k)$, which is necessary for allowability for $c \sigma$ with respect to $\bar{p}$. This argument also works if $k=n+1$ and $|\sigma| \cap y \neq \emptyset$. The only other case to check is when $k=n+1$ and $|\sigma| \cap y=\emptyset$. In this case $(c \sigma)^{-1}(y)$ is in the 0 skeleton of $c \sigma$, but we must have $0 \leq i+1-(n+1)+\bar{p}(n+1)$ since $\bar{p} \in J(i+1, n)$. Thus $c \sigma$ is allowable. Since this works for each $\sigma$ in $\xi$, we see that $c \xi$ is $J(i+1, n)$ allowable (and hence $J(i+2, n)$ allowable), and $\partial(c \xi)=\xi$, which was given as $J(i+1, n)$ allowable. Thus $c \xi \in I C_{i+1}^{J(n)}(c Y)$, and $I H_{*}^{J(n)}(c Y)=0$.

Now, suppose that $t<n$. In each of the allowability arguments above, we must replace $n$ with $t$. The first part of the argument goes through except for this cosmetic change. The only case that needs new verification is the case where $|\sigma| \cap y=\emptyset$. In this case we need $0 \leq i+1-(t+1)+\bar{p}(t+1)$. But we do know that $0 \leq i+1-(n+1)+\bar{p}(n+1)$, since $\bar{p} \in J(i+1, n)$, and so it suffices to have $-(t+1)+\bar{p}(t+1) \geq-(n+1)+\bar{p}(n+1)$, or $n+1-(t+1) \geq \bar{p}(n+1)-\bar{p}(t+1)$. But this is certainly true since $\bar{p}(k+1) \leq \bar{p}(k)+1$, by assumption for all $k$.

Before moving on to the next lemma, we observe that for $A$ and open subset of $X$, $I C_{*}^{J / n}(X, A)$ can be defined as the quotient of either inclusion $I C_{*}^{J(n)}(X, A) \hookrightarrow I C_{*}^{J}(X, A)$ or $I C_{*}^{J / n}(A) \hookrightarrow I C_{*}^{J / n}(X)$. In fact, we have the following commutative diagram of short exact sequences:

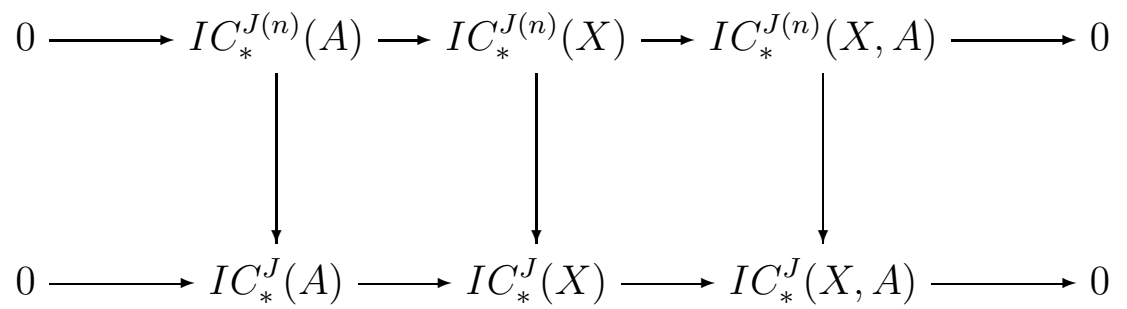


The vertical arrows readily can be checked to be injections, and so the snake lemma gives us the cokernel short exact sequence which is precisely

$$
0 \longrightarrow I C_{*}^{J / n}(A) \longrightarrow I C_{*}^{J / n}(X) \longrightarrow I C_{*}^{J / n}(X, A) \longrightarrow 0
$$

Lemma 5.2. Let $Y$ be a t-dimensional stratified space, and suppose $n \geq t$. Then $I H_{i}^{J / n}(c Y, Y)=$ 0 , and $I H_{i}^{J / n}(c Y) \cong I H_{i}^{J / n}(Y)$.

Proof. Consider the exact sequence

$$
\text { - } I H_{i}^{J / n}(Y) \longrightarrow I H_{i}^{J / n}(c Y) \longrightarrow I H_{i}^{J / n}(c Y, Y) \longrightarrow
$$

By Lemma 5.1, $I H_{i}^{J}(c Y) \rightarrow I H_{i}^{J / n}(c Y)$ is an isomorphism, and $I H_{i}^{J / n}(Y) \cong I H_{i}^{J}(c Y)$, by Proposition 3.2. These isomorphisms can be seen to commute (recalling that $J(i, n) \subset$ $J(i+1, n))$, and so $I H^{J / n}(Y) \rightarrow I H^{J / n}(c Y)$ is an isomorphism, proving the claim.

\section{Towards topological invariance?}

In this section, we outline a proposed proof of the topological invariance of $I H_{*}^{J}$ for locally conelike stratified spaces. This proposed proof follows the outline of King's proof in [5] for the single perversity case. We are currently unable to complete the proof at one step, but we will indicate how proving that step completes the whole proof. We will also show that topological invariance holds in a certain special case.

We begin by recalling the main ideas of King's proof from [5] of topological invariance of $I H_{*}^{\bar{p}}$. The idea is to start with the functor $X \rightarrow X^{*}$ taking a locally conelike stratified space (called a CS set in [5]) to the CS set that is topologically equivalent to $X$ but that is stratified with the coarsest intrinsic stratification. This functor yields maps $I H_{*}^{\bar{p}}(X) \rightarrow I H_{*}^{\bar{p}}\left(X^{*}\right)$, since the property $\bar{p}(k) \leq \bar{p}(k+1)$ implies that if a simplex is allowable in $X$ then it is also allowable in $X^{*}$. This will also be true for $I H_{*}^{J}(X)$. A CS set $x$ has property $\mathcal{H}$ if $I H_{*}^{\bar{p}}(X) \rightarrow I H_{*}^{\bar{p}}\left(X^{*}\right)$ is an isomorphism for all perversities $\bar{p}$.

The proof then inducts on depth, the difference in dimension between the highest and lowest dimensional non-empty strata of $X$. Let $P(i), Q(i)$, and $R(i)$ be the following statements:

$\mathbf{P}(\mathbf{i})$ All CS sets of depth $\leq i$ have property $\mathcal{H}$.

Q(i) All CS sets of the form $M \times c W, M$ a manifold, $W$ a compact filtered space, $\operatorname{depth}(W) \leq$ $i$ have property $\mathcal{H}$.

$\mathbf{R}(\mathbf{i})$ All CS sets of the form $\mathbb{R}^{k} \times c W, W$ a compact filtered space, $\operatorname{depth}(W) \leq i$ have property $\mathcal{H}$.

King goes on to show that $P(i)$ implies $R(i), R(i)$ implies $Q(i)$, and $P(i)$ and $Q(i)$ together imply $P(i+1)$. This implies the topological invariance of $I H_{*}^{\bar{p}}$ by induction, since $P(1)$ is clearly true. 
It is also clear that this framework would imply topological invariance of $I H_{*}^{J}$ if one could prove this sequence of implications with property $\mathcal{H}$ replaced by property $\mathcal{H}^{J}$ stating that $I H_{*}^{J}(X) \rightarrow I H_{*}^{J}\left(X^{*}\right)$ is an isomorphism for any $J$.

In fact, the implications $R(i)$ implies $Q(i)$, and $P(i)$ and $Q(i)$ together imply $P(i+1)$, as proven in [5], depend only on geometric properties of CS sets, the Mayer-Vietoris theorem, and the Künneth theorem of a CS set with a manifold. Since these properties all hold for $I H_{*}^{J}$, so do these implications. The problematic one is $P(i) \Rightarrow R(i)$, which relies on the actual cone formula properties of $I H^{\bar{p}}$.

The setting for attempting to prove $P(i)$ implies $R(i)$ is geometrically the following: We have a CS set $\mathbb{R}^{k} \times c W$, and by the arguments of Proposition 6 of $[5],\left(\mathbb{R}^{k} \times c W\right)^{*}=\mathbb{R}^{m} \times c Y$. We take $\operatorname{dim}(W)=s$ and $\operatorname{dim}(Y)=t$ so that $t=k-m+s$. Letting $h: \mathbb{R}^{k} \times c W \rightarrow \mathbb{R}^{m} \times c Y$ represent the (non-stratum-preserving) homeomorphism, it is also shown that $h^{-1}\left(\mathbb{R}^{m} \times *\right)=$ $\mathbb{R}^{k} \times c A$, where $A$ is an $m-k-1$ homology sphere and a union of components of strata of $W$. One also notes that $\mathbb{R}^{m} \times c Y-h\left(\mathbb{R}^{k} \times *\right)$, where $*$ is the cone point of $c W$, carries the intrinsic stratification of $\mathbb{R}^{k+1} \times W=\mathbb{R}^{k} \times(c W-*)$. Since each of these spaces has depth less than $\mathbb{R}^{k} \times c W$, they can be assume to have property $\mathcal{H}$ by induction. This is the basis for the remainder of the argument showing that $I H_{*}^{\bar{p}}\left(\mathbb{R}^{k} \times c W\right) \cong I H_{*}^{\bar{p}}\left(\mathbb{R}^{m} \times c Y\right)$, which is then based upon both some diagram chasing and some calculations involving the explicit cone formula for intersection homology with a single perversity. Unfortunately, points of this proof rely heavily on the precise formula - its vanishing in certain ranges and its equivalence to the intersection homology of the link in the other range. Thus any attempt to apply the overall argument to $I H_{*}^{J}$ must be modified.

We do not at present know how to show that $I H_{*}^{J}\left(\mathbb{R}^{k} \times c W\right) \cong I H_{*}^{J}\left(\mathbb{R}^{m} \times c Y\right)$, but we provide the partial result that $I H^{J}(c Y)$ is a direct summand of $I H^{J}(c W)$, under the induction hypothesis.

So, consider the space $c Y \times \mathbb{R}^{m}$, where $Y$ has dimension $t$. Let $t+m=n$, and let $y$ be the cone point of $y$. Let $V=c Y \times R^{m}-\mathbb{R}^{k} \times a$, where $a$ is the cone point of $c A$ so that $R^{k} \times a \subset y \times \mathbb{R}^{m}$. (Of course $\mathbb{R}^{k} \times a=\mathbb{R}^{k} \times w$, where $w$ is the cone point of $c W$, under the homeomorphism $h$ ).

Lemma 6.1. $I H_{i}^{J}(c Y)$ is a direct summand in $I H_{i}^{J / n}(V)$.

Note: no induction assumption is needed here.

Proof. Let $*$ be any point in $\mathbb{R}^{m}-\mathbb{R}^{k} \times a$ and let $\mathfrak{i}: c Y \times * \hookrightarrow V$ be the inclusion. Since this inclusion preserves codimensions of strata, it induces a map $\mathfrak{i}_{*}: I H_{*}^{J}(c Y) \rightarrow I H_{*}^{J}(V)$. This can be extended to a map $j_{*}: I H_{i}^{J}(c Y) \rightarrow I H_{i}^{J / n}(V)$ by composing $i_{*}$ with the natural $\operatorname{map} p_{*}: I H_{i}^{J}(V) \rightarrow I H_{i}^{J / n}(V)$.

We find a map that splits $j_{*}$. Note that we have a codimension preserving retraction $r: V \rightarrow c Y$ induced by projecting $c Y \times \mathbb{R}^{m}$ to $c Y \times *$. The retraction $r$ induces a map $r_{*}$ of all intersection homology groups of $V$ to those of $c Y$. In particular, we have $r_{*}$ : $I H_{i}^{J / n}(V) \rightarrow I H_{i}^{J / n}(c Y)$. We compose $r_{*}$ with $\rho_{*}: I H_{i}^{J / n}(c Y) \rightarrow I H_{i}^{J / t}(c Y)$, which exists since $J(i, t) \supset J(i, n)$ since $t \leq n$. But $I H_{i}^{J / t}(c Y)$ is isomorphic to $I H_{i}^{J}(c Y)$ by Lemma 5.1. Let's call this isomorphism $k_{*}: I H_{i}^{J / t}(c Y) \rightarrow I H_{i}^{J}(c Y)$. We claim that $\rho_{*} r_{*} j_{*} k_{*}$ is the identity on $I H_{i}^{J / t}(c Y)$, which implies that $k_{*} \rho_{*} r_{*}$ splits $j_{*}$. 
To verify the claim, we need only start with a cycle $\xi$ in $I C_{i}^{J / t}(c Y)$ and chase it through the maps. By the chain of isomorphisms $I H_{i}^{J / t}(Y) \cong I H_{i}^{J}(c Y) \cong I H^{J / t}(c Y)$ and their constructions in Proposition 3.2 and Lemma 5.1, it suffices to assume that $\xi=c \psi+\gamma$, where $\gamma$ is $J(i+1, t)$ allowable and supported in $Y \times \mathbb{R} \subset c Y$ and that $\psi$ is a $J(i, t)$ allowable $i-1$ chain, so that $c \psi$ is a $J(i, t)$ allowable $i$ chain. This chain suffices to represent both our given cycle in $I H_{i}^{J / t}(c Y)$ and its image under $k_{*}$ in $I H_{i}^{J}(c Y)$. Now, let's apply our other maps:

Under $\mathfrak{i}, \xi$ is simply included into $c Y \times * \subset V$. The map $p_{*}$ is induced from the quotient $I C_{i}^{J}(V) \rightarrow I C_{i}^{J / n}(V)$, so we can keep $\xi$ as a representative chain. The retract $r$ collapses $V$ back to $c Y \times *$, still not effecting our cycle, and finally $\rho_{*}$ is again induced by a quotient. Thus our representative cycle represents the same cycle after applying $\rho_{*} r_{*} j_{*} k_{*}$.

Corollary 6.2. If we assume inductively that $I H_{*}^{J}(W) \cong I H_{*}^{J}(V)$, then $I H_{i}^{J}(c Y)$ is a direct summand in $I H_{i}^{J / n}(W)$.

Proof. We have a commutative diagram

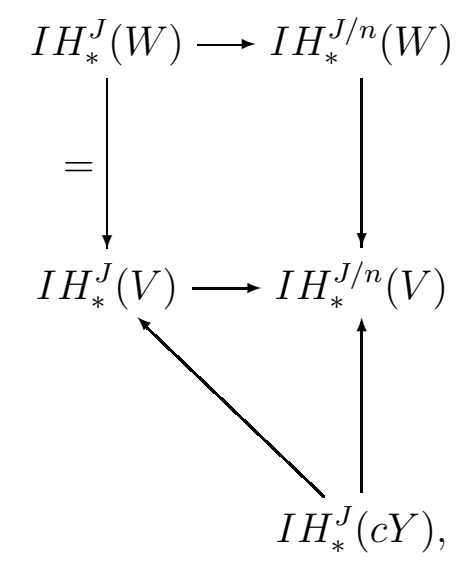

where the map $I H_{*}^{J}(c Y) \rightarrow I H_{*}^{J / n}(V)$ is a split injection by the preceding lemma and the diagonal map is is induced by inclusion. The corollary now follows by diagram chasing.

Remark 6.3. There are essentially two sticking points in finishing the proof of $P(i) \Rightarrow R(i)$ in the $J$ case. The first is to show that $I H_{*}^{J / n}(c Y) \cong I H^{J / n}(V)$, not just that it is a direct summand. The second is the show that if $I H_{*}^{J}(V) \cong I H_{*}^{J}(W)$, then $I H_{*}^{J / n}(V) \cong I H^{J / n}(W)$, or, equivalently, that if two spaces share the same intersection homology $I H_{*}^{J}$, then so do their cones.

Using Lemma 6.1, we can also prove the following basic case of invariance of $I H_{*}^{J}$ under restratification:

Proposition 6.4. Let $Y$ be a locally-conelike space of dimension $n-1$. Let $S Y$ be the suspension of $Y$. Then $I H_{*}^{J}(c S Y) \cong I H_{*}^{J}(c Y)$.

Proof. Of course as topological spaces (ignoring the stratifications), $c S Y \cong c Y \times \mathbb{R}$. The only difference in the stratifications of the two spaces is the existence of the extra cone point $v$ in the stratification of $c S Y$. 
Interpreting Lemma 6.1 in this situation, we see that $I H_{*}^{J}(c Y)$ (which is isomorphic to $I H^{J / n}(Y)$ by Lemma 5.1) is a summand in $I H_{*}^{J / n}(V)$, where $V=(c Y \times \mathbb{R})-v$. But $c Y \times \mathbb{R}-v$ has a stratum-preserving deformation retract to $(c Y \times-1) \cup(Y \times[-1,1]) \cup(c Y \times 1) \cong S Y$. So $I H_{*}^{J / n}(V) \cong I H_{*}^{J}(c S Y)$. So we just need to show that the summand injection is in fact an isomorphism, which will be the case if each homology class in $I H_{*}^{J / n}(V)$ has a representative with support in $c Y \times 1$

So suppose that $\xi$ is a cycle representing an element of $I H_{i}^{J / n}(V)$. If $|\xi| \cap \mathbb{R}^{-} \times y=\emptyset$, where $y$ is the cone point of $c Y$ and $\mathbb{R}^{-}=(0,-\infty)$, then we can use stratified homotopy invariance to push $\xi$ into $c Y \times 1$, whence $\xi$ represents an element of $I H_{i}^{J / n}(c Y)$. But if $|\xi| \cap R \times y \neq \emptyset$, then for each simplex $\sigma$ of $\xi$ with $\sigma \cap \mathbb{R}^{-} \times y \neq \emptyset$, if $\sigma$ is $\bar{p}$ allowable, then we must have $0 \leq i-n+\bar{p}(n)$, since $R^{-} \times y$ has codimension $n$ in $V$. So $\bar{p} \in J(i, n-1)$. Now we note that $J(i, n-1) \subset J(i+1, n)$ because $\bar{p}(n+1) \geq \bar{p}(n)$ for each perversity. So after subdividing and regrouping, we can write

$$
\xi=\zeta+\gamma
$$

where $|\zeta| \cap \mathbb{R}^{-} \times y=\emptyset$ and $\gamma \subset I C_{i}^{J(i+1, n)}(V)$. We cannot, however, say that $\gamma=0$ in $I C_{i}^{J / n}(V)$ because we do not know that $\partial \gamma$ is $J(i, n)$ allowable. We can, however, write

$$
\partial \gamma=\eta+\omega
$$

where $\eta$ consists of the simplices shared by $\partial \gamma$ and (negatively) $\partial \zeta$ and $\omega$ consists of simplices subdivided from $\partial \xi$. Note that this makes $\omega J(i, n)$ allowable since $\xi$ is a cycle in $I C_{i}^{J / n}(V)$. Furthermore, by applying the usual arguments (see [1] and the discussion above in the proof of Lemma 3.1), $\eta$ will be $J(i+1, n)$ allowable, since $\gamma$ is. Also, $|\eta| \cap \mathbb{R}^{-} \times y=\emptyset$.

Now, we do the following. By initial homotopies, we may assume that $|\xi| \subset(c Y \times$ 1) $\cup(Y \times[-1,1]) \cup(c Y \times-1)$. We can also assume to have subdivided sufficiently that $|\gamma| \subset c Y \times-1$ (if this is not possible, then $|\xi| \cap c Y \times-1=\emptyset$, and we are done already). Consider now

$$
\Gamma=(\gamma \times 1)-(\eta \times[-1,1])-(\gamma \times-1),
$$

where the second factor in each term refers to a chain in $\mathbb{R}$. In particular, $\eta \times[-1,1]$ is the product chain, triangulated compatibly with $\eta \times-1$ and $\eta \times 1 . \eta \times[-1,1]$ is also $J(i+1, n)$ allowable since $\eta$ is (see [2]). Thus $\Gamma$ is $J(i+1, n)$ allowable. Furthermore,

$$
\begin{aligned}
\partial \Gamma & =(\eta \times 1)+(\omega \times 1)-((\eta \times 1)-(\eta \times-1)+(\partial \eta \times[-1,1]))-((\eta \times-1)+(\omega \times-1)) \\
& =(\omega \times 1)-(\partial \eta \times[-1,1])-(\omega \times-1) .
\end{aligned}
$$

We observe that $\partial \eta=-\partial \omega$, and again we can assume from our chain division process that $\partial \omega$ inherits the allowability of $\omega$. Thus $\partial \omega$, and hence $\partial \eta \times[-1,1]$ are $J(i, n)$ allowable. Thus, overall, $\Gamma$ is $J(i+1, n)$ allowable and $\partial \Gamma$ is $J(i, n)$ allowable, i.e. $\Gamma=0 \in I C_{i}^{J / n}(V)$.

But now we can form $\xi^{\prime}+\Gamma$, where $\xi^{\prime}$ is the subdivision of $\xi$ that we have been using, and observe that the $\gamma \times-1$ terms cancel, leaving no simplices in $\xi^{\prime}+\Gamma$ that intersect $\mathbb{R}^{-} \times y$. So $\xi^{\prime}+\Gamma$ can be homotoped into $c Y \times 1$, and since $\xi^{\prime}+\Gamma$ and $\xi$ represent the same element of $I H_{i}^{J / n}(V)$, we see that $I H_{*}^{J / n}(c Y) \rightarrow I H_{*}^{J / n}(V)$ is indeed a surjection. 


\section{A sample computation}

In this section, we provide a sample calculation of $I H_{*}^{J}$ in a simple case. This is provided mainly to prove that $I H_{*}^{J} \not I H_{*}^{\bar{s}}$, where $\bar{s}$ is the perversity given by $\bar{s}(k)=\sup \{\bar{p}(k) \mid \bar{p} \in J\}$.

Let $X^{3}$ be a CS set of dimension 3 such that the singular set $\Sigma$ consists of a single point $x$, i.e., $X=X^{3} \supset X^{2}=X^{1}=X^{0}=x$. Let $\bar{p}$ be the perversity $(0,1,1,1)$ (beginning with $\bar{p}(2)=0)$, and let $\bar{q}$ be the perversity $(0,0,1,2)$. Let $J=\{\bar{p}, \bar{q}\}$. We will calculate $I H_{*}^{J}\left(c\left(X \times S^{1}\right)\right)$.

We begin by noting that the singular set of $X \times S^{1}$ is the single stratum $x \times S^{1}$, and it has codimension 3. Since $\bar{p}(3)>\bar{q}(3), I H_{*}^{J}\left(X \times S^{1}\right)=I H^{\bar{p}}\left(X \times S^{1}\right)$. This will be typical of a space $Z$ with only one singular stratum of codimension $k: I H^{J}(Z)$ will equal $I H_{*}^{\bar{r}}(Z)$, where $\bar{r}$ is any perversity in $J$ such that $\bar{r}(k) \geq \bar{u}(k)$ for all $\bar{u} \in J$.

Next we consider $I H_{*}^{J(4)}\left(X \times S^{1}\right)$. To compute these groups, we need to know what $J(i, 4)$ is for each $i$. Recall that $J(i, k)=\left\{\bar{p}_{j} \in J \mid 0 \leq i-(k+1)+\bar{p}_{j}(k+1)\right\}$. In this case, $J(i, 4)=\left\{\bar{p}_{j} \in J \mid 0 \leq i-5+\bar{p}_{j}(5)\right\}$. Thus $J(i, 4)$ contains $\bar{p}$ when $0 \leq i-4$, and it contains $\bar{q}$ when $0 \leq i-3$. In other words,

$$
\begin{aligned}
& J(0,4)=\emptyset \\
& J(1,4)=\emptyset \\
& J(2,4)=\emptyset \\
& J(3,4)=\bar{q} \\
& J(4,4)=J .
\end{aligned}
$$

Now, $I C_{i}^{J(4)}\left(X \times S^{1}\right)=\left\{\xi \in I C_{i}^{J}\left(X \times S^{1}\right) \mid \xi \in I C_{i}^{J(i+1,4)}\left(X \times S^{1}\right), \partial \xi \in I C_{i-1}^{J(i, 4)}\left(X \times S^{1}\right)\right\}$. So

$$
\begin{aligned}
& I C_{0}^{J(4)}\left(X \times S^{1}\right)=\emptyset \\
& I C_{1}^{J(4)}\left(X \times S^{1}\right)=\emptyset \\
& I C_{2}^{J(4)}\left(X \times S^{1}\right)=\left\{\xi \in I C_{2}^{\bar{q}}\left(X \times S^{1}\right) \mid \partial \xi=0\right\} \\
& I C_{3}^{J(4)}\left(X \times S^{1}\right)=\left\{\xi \in I C_{3}^{J}\left(X \times S^{1}\right)=I C_{3}^{\bar{p}}\left(X \times S^{1}\right) \mid \partial \xi \in I C^{\bar{q}}\left(X \times S^{1}\right)\right\} \\
& I C_{4}^{J(4)}\left(X \times S^{1}\right)=\left\{\xi \in I C_{4}^{J}\left(X \times S^{1}\right)=I C_{4}^{\bar{p}}\left(X \times S^{1}\right)\right\} .
\end{aligned}
$$

Thus,

$$
\begin{aligned}
& I H_{0}^{J(4)}\left(X \times S^{1}\right)=0 \\
& I H_{1}^{J(4)}\left(X \times S^{1}\right)=0 \\
& I H_{2}^{J(4)}\left(X \times S^{1}\right)=\operatorname{im}\left(I H_{2}^{\bar{q}}\left(X \times S^{1}\right) \rightarrow I H_{2}^{\bar{p}}\left(X \times S^{1}\right)\right) \\
& I H_{3}^{J(4)}\left(X \times S^{1}\right)=I H_{3}^{\bar{p}}\left(X \times S^{1}\right) \\
& I H_{4}^{J(4)}\left(X \times S^{1}\right)=I H_{4}^{\bar{p}}\left(X \times S^{1}\right) .
\end{aligned}
$$


From the long exact sequence for $I H_{*}^{J(n)}, I H_{*}^{J}$, and $I H_{*}^{J / n}$, and recalling $I H_{*}^{J}\left(X \times S^{1}\right)=$ $I H^{\bar{p}}\left(X \times S^{1}\right)$, we get

$$
\begin{aligned}
& I H_{0}^{J / 4}\left(X \times S^{1}\right)=I H_{0}^{\bar{p}}\left(X \times S^{1}\right) \\
& I H_{1}^{J / 4}\left(X \times S^{1}\right)=I H_{1}^{\bar{p}}\left(X \times S^{1}\right) \\
& I H_{2}^{J / 4}\left(X \times S^{1}\right)=\operatorname{cok}\left(I H_{2}^{\bar{q}}\left(X \times S^{1}\right) \rightarrow I H_{2}^{\bar{p}}\left(X \times S^{1}\right)\right) \\
& I H_{3}^{J / 4}\left(X \times S^{1}\right)=0 \\
& I H_{4}^{J / 4}\left(X \times S^{1}\right)=0,
\end{aligned}
$$

which are the groups of $I H_{*}^{J}\left(c\left(X \times S^{1}\right)\right)$ by the cone formula.

We note by contrast that the supremum perversity for $J$ is $\bar{s}=(0,1,1,2)$, and by the standard cone formula,

$$
I H_{i}^{\bar{s}}\left(c\left(X \times S^{1}\right)\right)= \begin{cases}0, & i \geq 2, \\ I H_{i}^{\bar{s}}\left(X \times S^{1}\right)=I H_{i}^{\bar{p}}\left(X \times S^{1}\right), & i<2 .\end{cases}
$$

These groups can well be different, as it is not difficult to find examples for which $\operatorname{cok}\left(I H_{2}^{\bar{q}}(X \times\right.$ $\left.\left.S^{1}\right) \rightarrow I H_{2}^{\bar{p}}\left(X \times S^{1}\right)\right) \neq 0$. For example, suppose that $X$ is the suspension of the torus $T^{2}$. Then by the Künneth theorem for products with a manifold and some elementary comutation of $I H_{*}(X)$, one has

$$
\begin{aligned}
& I H_{2}^{\bar{q}}\left(X \times S^{1}\right) \cong\left(I H_{2}^{\bar{q}}(X) \otimes H_{0}\left(S_{1}\right)\right) \oplus\left(I H_{1}^{\bar{q}}(X) \otimes H_{1}\left(S_{1}\right)\right) \cong(0 \otimes \mathbb{Z}) \oplus\left(\mathbb{Z}^{2} \otimes \mathbb{Z}\right) \cong \mathbb{Z}^{2} \\
& I H_{2}^{\bar{p}}\left(X \times S^{1}\right) \cong\left(I H_{2}^{\bar{p}}(X) \otimes H_{0}\left(S_{1}\right)\right) \oplus\left(I H_{1}^{\bar{p}}(X) \otimes H_{1}\left(S_{1}\right)\right) \cong\left(\mathbb{Z}^{2} \otimes \mathbb{Z}\right) \oplus(0 \otimes \mathbb{Z}) \cong \mathbb{Z}^{2} .
\end{aligned}
$$

These groups are isomorphic (in fact they must be, at least with rational coefficients, by the Goresky-MacPherson Poincaré Duality [3]). However, we observe that the generators of $I H_{2}^{\bar{q}}\left(X \times S^{1}\right)$ are simply the products of generators of $H_{1}\left(T^{2}\right)$ with $S^{1}$, e.g. each has the form $\xi \times S^{1}$. These terms goes to 0 in $I H_{2}^{\bar{p}}\left(X \times S^{1}\right)$, where they are bounded by chains of the form $\bar{c} \xi \times S^{1}$, where $\bar{c} \xi$ is the cone on $\xi$ to one "pole" of the suspension. Thus the homomorphism $I H_{2}^{\bar{q}}\left(X \times S^{1}\right) \rightarrow I H_{2}^{\bar{p}}\left(X \times S^{1}\right)$ is trivial, and the cokernel is $\mathbb{Z}^{2}$.

\section{Duality?}

A natural question to ask is whether $I H_{*}^{J}$ satisfies any form of Poincaré duality. We note that the most obvious thing to try does not work.

For a perversity, $\bar{p}$, let $\hat{p}$ be the dual perversity: $\bar{p}(k)+\hat{p}(k)=k-2$. Then it is natural to try to set $\hat{J}=\{\hat{p} \mid \bar{p} \in J\}$.

However, suppose that $X$ has only a point singularity of codimension $n$, that $J=\{\bar{p}, \bar{q}\}$ and that $\bar{p}(k)>\bar{q}(k)$. Then one we note that $I H_{*}^{J}(X ; \mathbb{Q})=I H_{*}^{\bar{p}}(X ; \mathbb{Q}) \cong I H_{n-*}^{\hat{p}}(X ; Q)$. But since $\bar{p}(k)>\bar{q}(k), \hat{q}(k)>\hat{p}(k)$ and $I H_{n-*}^{\hat{J}}(X ; \mathbb{Q})=I H_{n-*}^{\hat{q}}(X ; \mathbb{Q})$. So this is not the correct way to get duality. 


\section{Questions}

1. Is $I H_{*}^{J}$ a topological invariant?

2. If $\operatorname{dim} X=\operatorname{dim} Y$ and $I H_{*}^{J}(X) \cong I H_{*}^{J}(Y)$, does $I H_{*}^{J}(c X) \cong I H_{*}^{J}(c Y)$ ?

3. Can $I H^{J}$ always be calculated from knowledge of all $I H^{\bar{p}}$ ?

4. Does $I H_{*}^{J}$ possess a generalization of Poincaré duality?

5. Is there a "nice" formula for $I H_{*}^{J}(S X)$ in terms of $I H_{*}^{J}(X)$ ?

6. Is there a Deligne-type sheaf theoretic description of $I H_{*}^{J}$ ?

- If so, can it be axiomatized?

- Is there a duality theory via Verdier duality?

7. Is there a simple collection of spaces $\left\{X_{i}\right\}$ sufficient to determine $J$ given $I H_{*}^{J}\left(X_{i}\right)$ for all $i$ ?

8. What spaces are distinguished by $I H_{*}^{J}$ but not by any $I H_{*}^{\bar{p}}$ ?

9. Is there a simple collection of spaces $\left\{X_{i}\right\}$ which represents all possible functions from multiperversities $J$ to sequences of nonnegative integers $\left(\operatorname{dim} I H_{i}^{J}(X, \mathbb{Q}):=0,1, \ldots, n\right)$ for $n$-pseudomanifolds. (This question is of interest already for the usual intersection homology groups.)

10. Is it possible to prove PL-invariance of $I H_{*}^{J}(X)$ for simplicial pseudomanifolds $X$ based on the Goresky and MacPherson treatement of the behavior under subdivisions. (As far as we know, showing independence from the stratification along this line is not known even for the original intersection homology groups.)

Concerning Problem 7 note that for ordinary intersection homology groups, if we consider the set of open cones over products of spheres (or suspensions over products of spheres), then for an unknown perversity $\bar{p}$, given $I H_{i}^{\bar{p}}(X)$ for these spaces we can determine $\bar{p}$ uniquely. For $I H^{J}$ a larger family may be needed. The class of spaces containing spheres and closed under forming cones and taking Cartesian products may be relevant to problems 7 and perhaps also to Problems 8 and 9.

Finally we note that multiperversities may not be the end of the road. We can define more and more refined conditions on allowable singular chains by considering next chains in $I C^{J_{1}} \cap I C^{J_{2}} \cap \ldots$ for a set $\left\{J_{1}, J_{2}, \ldots\right\}$ of multiperversities. And continue by alternating between sums and intersections. We will not say more about this further generalization but rather introduce a notation: Tolerance will be a collective name for perversities, sets of perversities, sets of sets of perversities etc. (Thus, 0-tolerance is a single perversity.) Since duality appears to replace sums and intersections, duality which exists for 0-tolerance may be regained by taking tolerance to the limit. 


\section{References}

[1] Greg Friedman, Singular chain intersection homology for traditional and super-perversities, to appear in Trans. Amer. Math. Soc.; see also http://www.arxiv.org/abs/math.GT/0407301.

[2] _ Stratified fibrations and the intersection homology of the regular neighborhoods of bottom strata, Topology and Its Applications 134 (2003), 69-109.

[3] Mark Goresky and Robert MacPherson, Intersection homology theory, Topology 19 (1980), 135-162.

[4] _ Intersection homology II, Invent. Math. 72 (1983), 77-129.

[5] Henry C. King, Topological invariance of intersection homology without sheaves, Topology Appl. 20 (1985), 149-160.

[6] James R. Munkres, Elements of algebraic topology, Addison-Wesley, Reading, MA, 1984. 